\title{
Cell culture: building a better matrix
}

\author{
Nathan Blow
}

\begin{abstract}
With the realization that cells interact extensively with their surrounding microenvironments during growth and development, the challenge for researchers has become designing three-dimensional culture systems that more closely mimic those relationships.
\end{abstract}

When Matthias Lutolf from the Ecole Polytechnique Federale de Lausanne (EPFL) started looking at human stem cells under various culture conditions, something quickly caught his eye. "The biggest surprise for me was how different these cells are when grown in a three-dimensional environment," he recalls. "I realized then that we have many things to learn."

When it comes to understanding how stem cells interact with their surroundings, that learning may be just starting. In 2006, Dennis Discher from the University of Pennsylvania and his colleagues showed that the stiffness of a synthetic threedimensional extracellular matrix (ECM) could influence the differentiation of adult stem cells ${ }^{1}$. And more recently in June 2009, George Daley from Harvard Medical School and his colleagues writing in Nature and Leonard Zon, also from Harvard Medical School, and his colleagues writing in Cell reported that the development of hematopoietic stem cells is strongly influenced by the mechanical properties of the blood flow ${ }^{2,3}$. Although these studies focused mainly on mechanical interactions between cells and their microenvironments, it is well known that the various proteins and ligands contained within many of these environments can also influence the activities of surrounding cells.

"I think more and more people are realizing that growing cells on plastic is an artificial situation," says Jay George, chief technology officer at Trevigen. Although many researchers are starting to come to terms with this idea, in practice defining and replicating the natural microenvironment for a wide range of cell types in the laboratory or for use in tissue engineering applications is proving far from straightforward.

\section{Materials tool chest}

Structurally, ECMs are composed of proteins such as collagen and elastin that serve as scaffolds for cells, as well as networks of various adhesion ligands and growth factors, which promote cell signaling. Although ECM is complex in both structure and composition, it might surprise many biologists to learn just how far chemists and bioengineers have come in recent years in recreating parts of the ECM. "The stage [at which] materials exist today is very advanced," says Jeffrey Hubbell, a professor of bioengineering at the EPFL, "but the stage at which they have transitioned to everyday use in cell biology labs is not advanced at all."

In 2005, Hubbell wrote a review article in Nature Biotechnology suggesting the development of a 'toolkit' of synthetic biomaterials that could be used by scientists as building blocks to reconstruct various cellular microenvironments ${ }^{4}$. Today he says researchers and developers are quickly filling up that kit with proteins and small molecules-not to mention natural extract ECMs, engineered ECMs and even single protein ECMs.

Trevigen was founded in 1992 to explore the enzymology behind the processes of DNA damage and repair. As scientists at the company spent more time developing assays to study programmed cell death, along with other cellular behaviors, they increasingly saw the need for changing the way they were looking at cells. "For us at the time the next logical step was to start looking for a way to explore behavior in

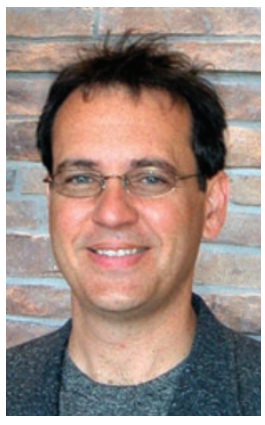

Jeff Hubbell is developing better extracellular matrices for cell culture and tissue engineering applications. culture such that the cells mimicked as close as possible the phenotypic properties of the tissue they came from," says Trevigen's George.

Initially, Trevigen focused on making different ECM proteins along with basement membrane extracts, which are largely comprised of laminins and collagens, on a large scale. Soon afterward they decided to integrate these extracts and proteins into their core cellular assays as well as develop new migration and invasion assays. In the end, they developed a culture system called Cultrex along with the kits for collecting and proliferating cells on three-dimensional matrices. "We are trying to integrate the matrix into as many assays as possible," notes George.

Trevigen is not alone in providing researchers natural ECM scaffolds and protein components upon which cells can be cultured. One of the earliest basement membrane extract formulations was Matrigel from BD Biosciences, a basement membrane extract of protein from mouse tumor cells, which is still used by many researchers today. It is similar to Millipore's ECMatrix. Other natural scaffolds have emerged as well, including different alginate-based systems such as AlgiMatrix from Invitrogen. And several other companies supply constituents of the ECM, such 
as purified collagen or laminin, for use in basic three-dimensional cell culturing applications.

Whereas basement membrane extracts provide a rich environment on which cells can be grown, some scientists have worked on advancing more defined self-assembling hydrogel formulations for threedimensional cell culture and tissue engineering applications. Glycosan BioSystems was founded in 2006 by Glenn Prestwich from the University of Utah to commercialize his research on hyaluronic acid, a carbohydrate that is a major component of the natural ECM, as a scaffold for cell culture and tissue engineering. Years of tinkering has led to a formulation in which thiol groups have been added to the hyaluronic acid, which allows a hydrogel to be formed in the absence of cross-linkers. But a crosslinking agent can also be used to provide greater control over matrix stiffness. Glycosan now offers HyStem for culturing stem cells and Extracel for general cell culture based on this hyaluronic acid hydrogel scaffold formulation.

As interest in developing hydrogels in which cells can be mixed with matrix before solidifying goes beyond the lab, many researchers see these gels as offering a bridge between culturing cells in the lab and tissue engineering applications in the clinic, albeit with some modifications. "For a lot of scientists who are doing basic research, these gels are wonderful," says Tom Zarembinski, director of business development at Glycosan BioSystems. "But for the other set with an eye towards translation, you also want something that is [US Food and Drug Administration] approvable, as well as providing a physiological milieu for the cells after transplantation." Zarembinski points out that several early hydrogel formulations are only liquid at $4{ }^{\circ} \mathrm{C}$ or at very low $\mathrm{pH}$, making them an inhospitable environment for most types of cells. But several gels have emerged that are well-defined, controllable and solidify under more physiological conditions. And researchers have even more options now as a new class of hydrogels whose properties can be dynamically controlled by light was recently described by Kristi Anseth and her colleagues in Science ${ }^{5}$. Using photactivable groups that were incorporated into the polymer backbone, the researchers demonstrated the ability to modulate the structural characteristics of their matrix even in the presence of cells without any adverse effects to those cells.

"I think the major challenge that we are finding is one hydrogel formulation does not fit all," says Zarembinski. Developers at Glycosan have worked with several collaborators, for whom culturing cells on basic hydrogel formulations provided a suitable substitute for proliferation. But then there have been some cell lines that need to be supplemented with key growth factors, clearly demonstrating the need for specialization.

Hubbell has been adapting a more common formulation for several of his tissue engineering efforts. "Fibrin can be purified from human blood plasma, and it is used as a surgical material already, so you are sort of adding onto something already available," he says. Hubbell notes that with fibrin they can take advantage of the enzymology of coagulation to incorporate engineered growth factor variants into the fibrin clot in a bound form or binding sites for growth or adhesion factors. And he notes that the cost of such a scaffold could potentially be much less than a synthetic matrix.

Although natural extracts and hydrogels provide a structure that can mimic the overall mechanics of many natural ECMs, according to Hubbell these scaffolds lack the fibrilular nature of in vivo ECMs, which is important for providing strength and tension to the scaffolds. "There are whole other classes of nonnatural gel- or peptidebased self-assembling gels that are fibrillar," he notes. One such material is 3DM's PuraMatrix which was developed in the laboratory of Shuguang Zhang at the Massachusetts Institute of Technology. Unlike the Glycosan's hyaluronic acid-based hydrogel, PuraMatrix is an amino acidbased hydrogel formulation consisting of 16-mer oligopeptides that self-assemble into nanofibers to form a matrix under physiological salt concentrations.

By providing so many options, Hubbell's toolkit might be one of the challenges when it comes to drawing more biologists to three-dimensional cell culture. "From the pragmatic perspective, the challenge is that there are so many things that you can vary now," says Hubbell, noting that with all the ECM analogs currently available, researchers can change matrix mechanics, the fibrin or gel nature of a matrix, as well as vary ligands and growth factors and their concentrations. "It is like buying a new stereo with a hundred knobs, and it can be overwhelming at first."

\section{One builder's story}

Lutolf at EPFL is straddling two worlds with his research. Coming from a bioengineering background, he decided to explore stem cell biology during a post-doc with Helen Blau at Stanford University. Now as he starts his own lab, Lutolf thinks his engineering skills could have a critical role helping him understand stem cell biology. "Signals are interpreted by the cell and transduced through complicated signaling networks, but ultimately these signals are coming

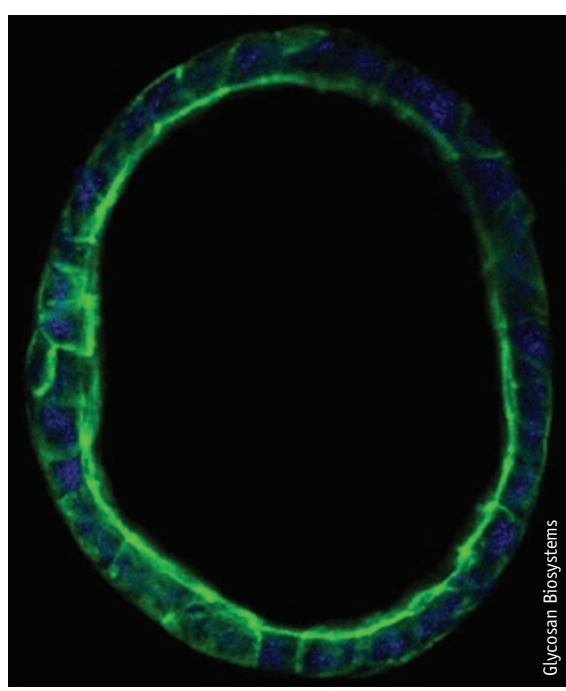

Madin-Darby canine kidney cells encapsulated in Glycosan Biosystems' HyStem-C hydrogel. 


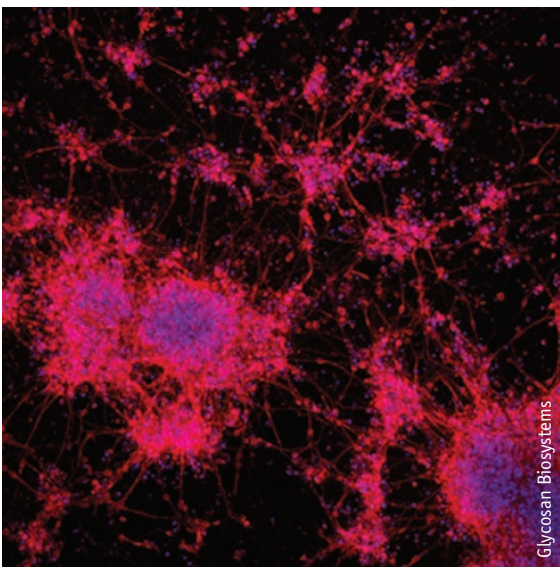

Human embryonic stem cell derived neruospheres grown with a three-dimensional cell culture system.

from the environment," he says, which is why he has focused his research efforts on recreating as many of these environments as possible trying to identify which ones could act as true stem cell niches.

Although Lutolf faces the challenge of recreating different microenvironments that could contain hundreds of proteins and sugars embedded in ECMs that possess different mechanical properties, he thinks there could be a way to get a handle on how these environments affect the properties of stem cells. "What we and others are trying to develop are new three-dimensional screening platforms where we can use physiological environments, but screened in a high-throughput fashion," he explains.

Starting with an inert, neutral polyethylene glycol (PEG) base, Lutolf's group is generating hundreds to thousands of miniaturized microenvironments by engineering bioactivity onto their PEG scaffolds and looking at cell responses. "We have solutions of liquid polymers that can be cross-linked under physiological conditions to form solid hydrogels," says Lutolf. Using robots, these hydrogels, which when spotted are only 50 to 100 nanoliters, with their incorporated stem cells can be arrayed on glass slides for testing. His system has the ability to vary the ECM stiffness by adding growth factors, proteins and peptide ligands. Initial experiments have focused on 5-10 different ECM stiffnesses with up 20 different signaling molecules.
The real challenge with such a highthroughput approach is being able to look at how the cells are reacting to life in so many different microenvironments. Currently Lutolf is using an in situ readout for the state of his stem cells in culture. Using engineered stem cell lines containing green fluorescent protein, the group can tell whether the stem cells are in a self-renewing state or not. Applying automated microscopy to individual spots gives a real-time picture of cell activity. But real-time imaging of cells in this world does come at a price.

"Three dimensional structures are more complicated; multiple sections have to be imaged," says Lutlof, which takes up a tremendous amount of computer storage space.

Other developers are also looking to offer high-throughput screening solutions designed for optimizing three-dimensional cell culture. "We are working on a microdroplet screening array where each hydorgel droplet has a different composition of gel, so researchers can screen on the microarray and with their cells to see which droplets elicit the desired response," says Glycosan's Zarembinski.

As chemists and bioengineers push the boundaries of three-dimensional cell culture, the question of when, or if, biologists will fully embrace these approaches remains. But if biologists are interested in mimicking the natural world more closely in their cell culture experiments, George thinks there is no other way. "Cells normally do not grow in plastic. I do not know of a plastic organism.”

1. Engler, J.A., Sen, S., Sweeney, H.L. \& Discher, D.E. Matrix elasticity direct stem cell lineage specification. Cell 126, 677-689 (2006).

2. Adamo, L. et al. Biomechanical forces promote embryonic haematopoiesis. Nature 459, 11311134 (2009).

3. North, T.E. et al. Hematopoietic stem cell development is dependent on blood flow. Cell 137, 736-748 (2009).

4. Lutolf, M.P. \& Hubbel, J.A. Synthetic biomaterials as instructive extracellular microenvironments for morphogenesis in tissue engineering. Nat. Biotechnol. 23, 47-55 (2005).

5. Kloxin, A.M., Kasko, A.M., Salinas, C.N., \& Anseth, K.S. Photodegradable hydrogels for dynamic tuning of physical and chemical properties. Science 324, 59-63 (2009).

Nathan Blow is the technology editor for Nature and Nature Methods (n.blow@boston.nature.com). 


\section{SUPPLIERS GUIDE: COMPANIES OFFERING CELL CULTURE}

SOLUTIONS

Company

3DM

3D Biotek

Accuri Cytometers

Agilent Technologies

Andor Technology

Apogee Instruments

Applied BioPhysics

Applied Cytometry

Applied Scientific Instrumentation

Beckman Coulter

BectonDickinson

BioCat GmbH

Biomedical Photometrics

Bio-Rad

Cambio

Cell Biolabs, Inc.

Chroma Technology

Clontech

Dojindo

eMouseHouse

FiberCell Systems

Fujifilm

Glen Mills

Glycosan BioSystems

Guava Technologies

Harvard Apparatus

Ibidi

Inbiolabs

Innovative Cell Technologies

Intelligent Substrates

Invitrogen

Lampire Biological Products

Leica Microsystems

Millipore

Molecular Devices

MP Biomedicals

Nanoptek

Neuro Probe

Nikon Instruments

Olympus

Omega Optical

Perkin Elmer Life Sciences

Princeton Instruments

QImaging

Sigma Aldrich

Synthecon

Trevigen

Zeiss
Web address

http://www.puramatrix.com/

http://www.3dbiotek.com/

http://www.accuricytometers.com/

http://www.agilent.com/

http://www.andor-tech.com/

http://www.ccd.com/

http://www.biophysics.com/

http://www.appliedcytometry.com/

http://www.asiimaging.com/

http://www.beckmancoulter.com/

http://www.bd.com/

http://www.biocat.de/

http://www.genefocus.com/

http://www.bio-rad.com/

http://www.cambio.co.uk/

http://www.cellbiolabs.com/

http://www.chroma.com/

http://www.clontech.com/

http://www.dojindo.com/

http://www.emousehouse.com/

http://www.fibercellsystems.com/

http://www.fujimed.com/

http://www.glenmills.com/

http://www.glycosan.com/

http://www.guavatechnologies.com/

http://www.harvardapparatus.com/

http://www.ibidi.com/

http://www.inbiolabs.com/

http://www.innovativecelltech.com/

http://www.intelligentsubstrates.com/

http://www.invitrogen.com/

http://www.lampire.com/

http://www.leicamicrosystems.com/

http://www.millipore.com/

http://www.moleculardevices.com/

http://www.mpbio.com/

http://www.nanoptek.com/

http://www.neuroprobe.com/

http://www.nikoninstruments.com/

http://www.olympusamerica.com/

http://www.omegafilters.com/

http://las.perkinelmer.com/

http://www.princetoninstruments.com/

http://www.qimaging.com/

http://sigmaaldrich.com/

http://www.synthecon.com/

http://www.trevigen.com/

http://www.zeiss.com/ 